\title{
Observations of 54 Active Galactic Nuclei with the HEGRA system of Cherenkov telescopes
}

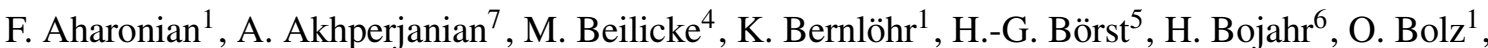 \\ T. Coarasa ${ }^{2}$, J. L. Contreras ${ }^{3}$, J. Cortina ${ }^{2}$, S. Denninghoff ${ }^{2}$, V. Fonseca ${ }^{3}$, M. Girma ${ }^{1}$, N. Götting ${ }^{4}$, \\ G. Heinzelmann ${ }^{4}$, G. Hermann ${ }^{1}$, A. Heusler ${ }^{1}$, W. Hofmann ${ }^{1}$, D. Horns ${ }^{1}$, I. Jung ${ }^{1}$, R. Kankanyan ${ }^{1}$, \\ M. Kestel ${ }^{2}$, A. Konopelko ${ }^{1}$, H. Kornmeyer ${ }^{2}$, D. Kranich ${ }^{2}$, H. Lampeitl ${ }^{1}$, M. Lopez ${ }^{3}$, E. Lorenz ${ }^{2}$, \\ F. Lucarelli ${ }^{3}$, O. Mang', D. Mazin ${ }^{4,10}$, H. Meyer 6 , R. Mirzoyan ${ }^{2}$, A. Moralejo ${ }^{3}$, E. Ona-Wilhelmi ${ }^{3}$, \\ M. Panter ${ }^{1}$, A. Plyasheshnikov ${ }^{1,8}$, G. Pühlhofer ${ }^{1}$, R. de los Reyes ${ }^{3}$, W. Rhode ${ }^{6}$, J. Ripken ${ }^{4}$, G. Rowell ${ }^{1}$, \\ V. Sahakian ${ }^{7}$, M. Samorski ${ }^{5}$, M. Schilling ${ }^{5}$, M. Siems ${ }^{5}$, D. Sobzynska ${ }^{2,9}$, W. Stamm ${ }^{5}$, \\ M. Tluczykont ${ }^{4,11}$, V. Vitale ${ }^{2}$, H. J. Völk ${ }^{1}$, C. A. Wiedner ${ }^{1}$, and W. Wittek ${ }^{2}$ \\ 1 Max-Planck-Institut für Kernphysik, Postfach 103980, 69029 Heidelberg, Germany \\ 2 Max-Planck-Institut für Physik, Föhringer Ring 6, 80805 München, Germany \\ 3 Universidad Complutense, Facultad de Ciencias Físicas, Ciudad Universitaria, 28040 Madrid, Spain \\ ${ }^{4}$ Universität Hamburg, Institut für Experimentalphysik, Luruper Chaussee 149, 22761 Hamburg, Germany \\ 5 Universität Kiel, Institut für Experimentelle und Angewandte Physik, Leibnizstraße 15-19, 24118 Kiel, Germany \\ ${ }^{6}$ Universität Wuppertal, Fachbereich Physik, Gaußstr. 20, 42097 Wuppertal, Germany \\ 7 Yerevan Physics Institute, Alikhanian Br. 2, 375036 Yerevan, Armenia \\ 8 On leave from Altai State University, Dimitrov Street 66, 656099 Barnaul, Russia \\ 9 Home institute: University Lodz, Poland \\ 10 Now at (2) \\ 11 Now at École Polytechnique, LLR, 91128 Palaiseau cedex, France
}

Received 28 November 2003 / Accepted 24 March 2004

\begin{abstract}
A sample of 54 selected Active Galactic Nuclei (AGN) has been observed with the HEGRA stereoscopic system of Cherenkov Telescopes between 1996 and 2002 in the TeV energy regime. The observations were motivated by the positive results obtained for Mkn 421 and Mkn 501. The distances of the selected objects vary over a large range of redshifts between $z=0.004$ and $z=0.7$. Among the observed AGN are the now-established TeV-emitting BL Lac type objects H 1426+428 and 1ES 1959+650. Furthermore the BL Lac object 1ES 2344+514 and the radio galaxy M 87 show evidence for a signal on a $4 \sigma$ level. The observation of 1ES 2344+514 together with the Whipple results firmly establishes this AGN as a TeV source. Several objects (PKS 2155-304, BL Lacertae, 3C 066A) that have been claimed as TeV $\gamma$-ray emitters by other groups are included in this data sample but could not be confirmed using data analysed here. The upper limits from several AGN included in this analysis are compared with predictions in the frame-work of SSC models.
\end{abstract}

Key words. gamma rays: observations - galaxies: active - galaxies: BL Lacertae objects: individual: 1ES 2344+514 BL Lacertae objects: individual: 1ES 1959+650 - BL Lacertae objects: individual: H 1426+428 - galaxies: individual: M 87

\section{Introduction}

In the commonly adopted view the "central engine" of Active Galactic Nuclei (AGN) consists of a super-massive black hole of up to $10^{9} M_{\odot}$ surrounded by an accretion disk. Two relativistic plasma outflows (jets) perpendicular to the accretion disk can be observed in some AGN (Rees 1984; Urry \& Padovani 1995). So far, $\gamma$-rays from AGN in the TeV energy regime have

Send offprint requests to: $\mathrm{M}$. Tluczykont, e-mail: Martin.Tluczykont@desy.de essentially been detected from objects of the BL Lac type, i.e. AGN having their jet pointing close to the observer's line of sight. Furthermore, all known TeV blazars are X-ray selected BL Lac objects. Recently, the first detection of $\mathrm{TeV} \gamma$-rays from the radio galaxy M 87 with the HEGRA Cherenkov telescopes was reported (Aharonian et al. 2003b).

Different models for the production of $\mathrm{TeV} \gamma$-rays from BL Lac objects have been proposed. In leptonic models the IC mechanism is assumed to produce the $\mathrm{TeV}$ emission (e.g. Sikora 2001), whereas in hadronic models the $\gamma$-rays are 
produced via the interactions of relativistic protons with matter (e.g. Pohl \& Schlickeiser 2000), ambient photons (Mannheim 1993) or magnetic field (Aharonian 2000), or both (Mücke \& Protheroe 2001).

The observed $\mathrm{TeV}$ emission shows high flux-variability on timescales stretching from months to less than an hour. Detailed studies of variability of BL Lac type objects can contribute to the understanding of their intrinsic acceleration mechanisms (Krawczynski et al. 2001; Aharonian et al. 2002a). The positive results obtained from the observations of the prominent extragalactic sources of TeV $\gamma$-rays Mkn 421 (Punch et al. 1992; Petry et al. 1996; Piron et al. 2001) and Mkn 501 (Quinn et al. 1996; Bradbury et al. 1997; Djannati-Ataï et al. 1999) as well as their relevance in relation to the question of the extragalactic background photon field (Aharonian 2001), have motivated further observations of Active Galactic Nuclei with the HEGRA Cherenkov telescopes. In this paper we present the results of dedicated observations of 54 AGN in the years 1996 to 2002 with the stereoscopic system of Cherenkov telescopes. After a brief introduction to the HEGRA Cherenkov telescopes the analyzed data-set will be presented, followed by a description of the analysis used in this paper and a presentation of the results. The paper closes with a discussion and a summary.

\section{The HEGRA Cherenkov telescope system}

The HEGRA stereoscopic Cherenkov telescope system (1996-2002) consisted of 5 imaging air Cherenkov telescopes (IACTs) (Daum et al. 1997) used in the stereoscopic observation mode on the Canary island of La Palma $\left(28.75^{\circ} \mathrm{N}\right.$, $17.90^{\circ} \mathrm{W}$ ) at an altitude of $2200 \mathrm{~m}$ a.s.l. Additionally, one telescope (not used for the present analysis) was operated in standalone mode (Mirzoyan et al. 1994). Each system telescope was equipped with an $8.5 \mathrm{~m}^{2}$ tesselated mirror dish of 30 single mirrors with a diameter of $60 \mathrm{~cm}$ each, and a camera consisting of 271 photomultiplier tubes (pixels). The HEGRA IACT system operated at an energy threshold of $0.5 \mathrm{TeV}$ for photons of vertical incidence, with energy and angular resolution of $\Delta E / E=10-20 \%$ and $0.1^{\circ}$ respectively on an event-byevent basis. The field of view of each system telescope had a diameter of $4.3^{\circ}$. The introduction of the stereoscopic observation technique results in an improvement of the sensitivity of Cherenkov telescopes and especially allows for an effective $\gamma$-hadron separation (see below). The performance of the HEGRA system of Cherenkov telescopes can be found in Pühlhofer et al. (2003).

\section{Data set}

Observations of 54 objects of the AGN class were carried out from 1996 to 2002, resulting in a total pre-selection exposure time of approx. $1150 \mathrm{~h}$ (not including Mkn 421 and Mkn 501) corresponding to more than one year of continuous observations in moonless nights with the HEGRA IACT system. The total observation time accumulated for Mkn 421 and Mkn 501 amounts to more than $1500 \mathrm{~h}$. The results of the HEGRA observations from these two objects were presented in different publications (Bradbury et al. 1997; Aharonian et al. 1999a,b,c; Sambruna et al. 2000; Aharonian et al. 2001a,b, 2002a) and are not included in the present work.

Mkn 421 and Mkn 501 excluded, the data set contains 37 objects identified and confirmed as BL Lac type objects, 10 radio galaxies, 4 Seyfert galaxies, 1 quasar and 2 galaxies following the catalogues of Stickel et al. (1994); Padovani \& Giommi (1995); Véron-Cetty \& Véron (2001). The object 1ES $0806+524$ is one of the BL Lac objects which was proposed by Tinyakov \& Tkachev (2001) to coincide with an AGASA UHECR triplet, thus being a candidate for the acceleration of ultra high energy cosmic rays. The distances of the objects vary over a large range of redshifts between $z \approx 0.004$ (M 87) and $z \approx 0.7$ (PKS 0219-164). However, the expected absorption due to pair production of $\mathrm{TeV} \gamma$-rays with the extragalactic background light (EBL) (Nikishov 1962) $\left(\gamma_{\mathrm{TeV}} \gamma_{\mathrm{EBL}} \rightarrow \mathrm{e}^{+} \mathrm{e}^{-}\right)$increases for larger redshifts, thus decreasing the detectability of objects located at large redshifts. Therefore, most of the observed objects were chosen with low redshift (i.e. $z<0.2)$.

All observations were carried out in the "wobble" observation mode (Aharonian et al. 1997), tracking the telescopes with an offset of $0.5^{\circ}$ in declination with respect to the object position, allowing for simultaneous on- and off-source (background) observations. For consecutive runs with a duration of $20 \mathrm{~min}$, the offset sign is reversed in order to avoid systematic effects due to acceptance inhomogeneities in the field of view. The background is estimated (similarly to the method used in Aharonian et al. 2003b) using a ring segment concentric to the camera center at the same radial distance to the camera center as the on-source region (i.e. $0.5^{\circ}$ ). A segment with opening angle $\eta=70^{\circ}$ is excluded from the background region in order to avoid possible contamination from the on-source region. This method makes sure that on- and off-source measurements are both taken with identical radial camera acceptances and allows simultaneously large background statistics.

Two a priori cuts on the system trigger rate are applied to each run in order to exclude runs taken under bad weather conditions and to reduce systematic effects in the determination of the excess rate. In a first step a minimum trigger rate of $7 \mathrm{~Hz}$ is required. This cut excludes data taken under the worst weather conditions. In a second step, an expected rate, depending on the hardware settings and the zenith angle of the observations is calculated from the parameters of a fit to all data of one period with constant hardware settings (all runs with trigger rate lower than $7 \mathrm{~Hz}$ are excluded from this fit). Runs with rates below $80 \%$ of the expected rate are rejected. Additionally, runs with technical problems are excluded. After the application of the above run-selection criteria the total cleaned data set yields an observation time of $1017 \mathrm{~h}$. In Table 4, all observed objects are listed with J2000 coordinates, redshift and object type, ordered by ascending redshift. Additionally, the observation time spent on each object as well as the results of the analysis described below are listed. 


\section{Data analysis}

The Cherenkov light generated by an air shower initiated by a primary $\gamma$-ray or hadronic particle is seen as an elliptical image in each triggered camera. Since each telescope has a different viewing angle relative to the shower axis a complete geometrical reconstruction of the air shower is possible with an image analysis of at least two telescopes.

Before the reconstruction of direction and shower core position the following cuts are applied. Reconstructed images with more than 15 defective camera pixels are rejected. A minimum amount of light (size) of 40 photo-electrons (ph.e.) is required in an image. Images with a distance of the center of gravity to the camera center of more than $78 \%$ of the camera radius $\left(4.3^{\circ}\right)$ are rejected in order to avoid truncation by the camera border.

After application of the above image selection criteria at least three remaining images are required in this analysis for the reconstruction of the direction and the core impact position of an event. This improves the quality of the reconstruction, the angular resolution and the separation between $\gamma$-ray and cosmic ray (hadronic background) induced air showers. The stereoscopic technique allows for an event-by-event reconstruction of the direction of the primary particle. Since the shape of the elliptical images also depends on the shower core position, the reconstruction of the shower impact parameter for each telescope provides a means of scaling the individual widths of the elliptical shower images of each telescope with expected widths for $\gamma$-ray induced shower images from Monte-Carlo simulations. The mean of the scaled widths is called mscw-parameter and provides a very good $\gamma$-hadron separation. This is described in detail in Konopelko et al. (1999a). The optimum cut value for a $\gamma$-ray signal search is found to be $\mathrm{mscw}=1.1$.

For the reconstruction of the direction of the primary particle, algorithm \# 3 from Hofmann et al. (1999) is used. The angle $\Delta \Theta=\left|\Theta_{0}-\Theta_{\mathrm{r}}\right|$ between the object direction $\Theta_{0}$ and the reconstructed shower axis $\Theta_{\mathrm{r}}$ is called the angular distance. In case of a signal from a source with point-like emission, small values of the squared angular distance $\Delta \Theta^{2}$ starting from 0 are expected to accumulate entries in the signal region. The extension and shape of the signal distribution in $\Delta \Theta^{2}$ reflects the angular resolution of the system and depends on the telescope multiplicity, the zenith angle and the hardware setup of the telescope system. Therefore, the cut on the angular distance also depends on the parameters mentioned above. Using data from the well-studied Crab Nebula, the cut on $\Delta \Theta^{2}$ is thus optimized individually for different hardware setups, multiplicities and zenith angle intervals. This method takes the dependencies of the angular resolution described above into account and leads to results consistent with earlier analyses. Typical values of the $\Delta \Theta^{2}$-cut are $0.008 \mathrm{deg}^{2}$ for events reconstructed with five triggered telescopes (having the best angular resolution) to $0.015 \mathrm{deg}^{2}$ for 3 -telescope events. Similarly, the cut on the core impact position slightly depends on the zenith angle (ZA) of the observation. The optimum values found for this cut are $200 \mathrm{~m}$ (low ZA), $400 \mathrm{~m}$ (medium ZA) and $600 \mathrm{~m}$ (high ZA). In Table 1 , all selection criteria and cuts are summarized.
Table 1. Selection criteria of the analysis chain. The cuts were optimized individually for all data subsets using data of the well-studied Crab Nebula (see text). The distance is measured from the center of gravity of the image to the camera center. The entry " $f$ (subset)" indicates that the cut depends on the data subset.

\begin{tabular}{l|ll}
\hline \hline \multirow{3}{*}{ Run selection } & Rate & $>7 \mathrm{~Hz}$ \\
& Rate deviation & $<20 \%$ \\
& Technical problems & - \\
\hline \multirow{3}{*}{ Image selection } & \# of defective pixels & $<15$ \\
& Image size & $>40$ ph.e. \\
& Distance & $<0.78$ \\
\hline \multirow{3}{*}{ Event selection } & Telescope multiplicity & $\geq 3$ \\
& Core distance & $<\mathrm{f}$ (subset) \\
& mscw & $<1.1$ \\
& $\Delta \Theta^{2}$ & $<\mathrm{f}$ (subset) \\
\hline
\end{tabular}

Different cuts on $\Delta \Theta^{2}$ imply different solid angle ratios of on- and off-source region $\alpha=\Omega_{\text {on }} / \Omega_{\text {off }}$ ( $\alpha$-factor) for each subset. Therefore the significance of an excess is calculated using a formula based upon the likelihood Eq. (17) of Li \& Ma (1983) but generalized for data subsets with different $\alpha$-factors ${ }^{1}$ :

$$
\begin{aligned}
\mathrm{S}= & \sqrt{2} \times\left[\sum_{i} N_{\mathrm{on}}^{(\mathrm{i})} \ln \left(\frac{\sum_{i} N_{\mathrm{on}}^{(\mathrm{i})}}{\sum_{i} \frac{\alpha_{i}}{1+\alpha_{i}}\left(N_{\mathrm{on}}^{(\mathrm{i})}+N_{\mathrm{off}}^{(\mathrm{i})}\right)}\right)\right. \\
& \left.+\sum_{i} N_{\mathrm{off}}^{(\mathrm{i})} \ln \left(\frac{\sum_{i} N_{\mathrm{off}}^{(\mathrm{i})}}{\sum_{i} \frac{1}{1+\alpha_{i}}\left(N_{\mathrm{on}}^{(\mathrm{i})}+N_{\mathrm{off}}^{(\mathrm{i})}\right)}\right)\right]^{1 / 2} .
\end{aligned}
$$

The variability of each object is investigated using the Kolmogorov and the Prahl test (Kolmogorov 1933; Prahl 1999). Both tests result in a significance for burst-like behaviour, given a time sequence of events. The Prahl test is especially sensitive to burst-like behaviour with a small duty cycle.

For each object a Crab Nebula $\gamma$-rate as expected for identical observational conditions (zenith angle, hardware setup) is calculated from data. These expected rates are used to compute flux values and upper limits on the integral flux following Helene (1983).

\section{Results}

A distribution of the significances for steady state emission (DC) of all analyzed objects is shown in Fig. 1. The distribution follows a Gaussian distribution of mean zero and standard deviation one, as expected in case of a pure background

\footnotetext{
${ }^{1}$ In Li \& Ma (1983) the significance is derived from the ratio of the conditional probabilities for "background assumption" and "signal assumption". Substituting both assumptions with a sum over data subsets with different $\alpha$-factors and a straight forward calculation leads to the above generalized formula.
} 


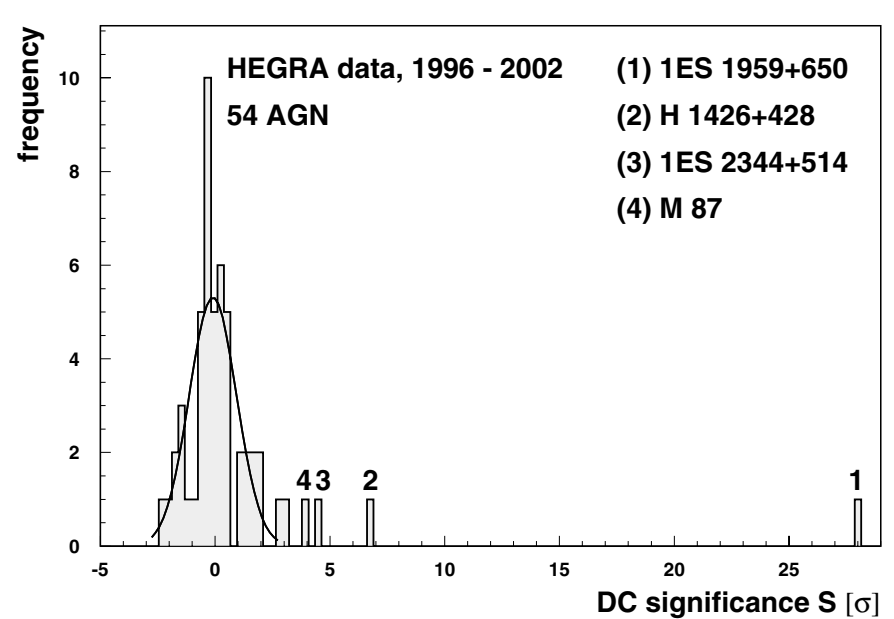

Fig. 1. Distribution of significances for all 54 objects analyzed in this work. A Gaussian fit between -2.5 and $+2.5 \sigma$ matches the core distribution very well. The objects 1 ES $1959+650$ and $\mathrm{H} 1426+428$ show a clear deviation from the background expectation. Two further objects, 1ES 2344+514 and M 87, also show a deviation from the background expectation on the $4 \sigma$ level and thus evidence for the emission of $\mathrm{TeV} \gamma$-radiation.

sample, with exceptions for 1ES 1959+650, H 1426+428, 1ES 2344+514 and M 87. In Table 4, a list of all objects analyzed in this work ordered by ascending redshift is shown along with their observation time and upper limits on the integral flux respectively flux values for the most significant objects. The values of the calculated upper limits are found to lie between $2 \%$ and $60 \%$ of the Crab Nebula flux. Comparable upper limits for BL Lac objects were found by observations of the Whipple telescope, ranging from $6 \%$ to $100 \%$ of the Crab Nebula flux (Horan et al. 2003; de la Calle Perez et al. 2003). The statistical tests on burst-like variability of all objects yield no positive results except for 1ES 1959+650 where a high statistical significance $(>16 \sigma)$ for burst-like behaviour reflects the strong obvious flaring activity in May and July 2002.

The X-ray selected BL Lac object 1ES 1959+650 (Elvis et al. 1992; Schachter et al. 1993) was first reported as a $\mathrm{TeV} \gamma$-ray emitter by the Seven Telescope Array group in 1999 with a DC significance of $3.9 \sigma$ (Nishiyama et al. 1999). HEGRA IACT system observations were carried out from July to September 2000, May to October 2001 and May to September 2002. The HEGRA results on 1ES 1959+650 were published in detail elsewhere (Aharonian et al. 2003c), the results presented in this analysis show a level of activity ranging from 0.06 to 2.9 Crab units and are consistent with the earlier analysis.

H 1426+428 was reported to have a synchrotron peak lying near or above $100 \mathrm{keV}$ (Costamante et al. 2001) thus qualifying the object as an extreme synchrotron blazar. Detections in the $\mathrm{TeV}$ energy regime from this object have been reported by the Whipple collaboration (Horan et al. 2002) the HEGRA collaboration (Aharonian et al. 2002b) and the CAT collaboration (Djannati-Ataï et al. 2002). The observations of H 1426+428 in the years 1999, 2000 and 2002 resulted in an excess on the
Table 2. Number of on- and off-source events and significance $S$ for the data sets from the years 1997 (P1), 1998 (P2) and 2002 (P3) on $1 \mathrm{ES} 2344+514$. Note that the major part of the excess is accumulated in 1998.

\begin{tabular}{llrrrc}
\hline \hline Observation periods & $\begin{array}{c}\text { Time } \\
{[\mathrm{h}]}\end{array}$ & $\begin{array}{r}N_{\text {on }} \\
\text { \# }\end{array}$ & $\begin{array}{r}\alpha N_{\text {off }} \\
\text { N }\end{array}$ & {$[\sigma]$} \\
\hline P1 & Oct.-Dec. 1997 & 15.0 & 54 & 52 & 0.3 \\
P2 & Aug.-Nov. 1998 & 41.8 & 128 & 84 & 4.3 \\
P3 & Sep. 2002 & 15.7 & 53 & 35 & 2.6 \\
\hline$\Sigma$ & & $\mathbf{7 2 . 5}$ & $\mathbf{2 3 5}$ & $\mathbf{1 7 1}$ & $\mathbf{4 . 4}$ \\
\hline
\end{tabular}

$7.5 \sigma$ level (Aharonian et al. 2003a), consistent with the analysis presented here $(6.6 \sigma)$.

1ES 2344+514 was one of the first BL Lac type objects to be reported as an extreme synchrotron blazar with synchrotron peak energy reaching up to $100 \mathrm{keV}$ (Giommi et al. 2000). The first $\mathrm{TeV}$ detection of this object was reported by the Whipple group in 1998 (Catanese et al. 1998). With an average flux of $11 \%$ of the Crab Nebula flux in 1998 and a higher flux level of $63 \%$ of the Crab flux in one night of observations $(6 \sigma)$, the object has shown clear evidence for a variable flux in the Whipple data. The results of the HEGRA observations on 1ES 2344+514 of the year 1997 and 1998 were first reported by Konopelko et al. (1999b) with a DC significance of $3.3 \sigma$. Further observations have been carried out since the above publication. The analysis presented here includes the complete dataset and results in an excess of $64 \pm 15$ photons $\left(N_{\text {on }}=235,\left\langle N_{\text {off }}\right\rangle=171\right)$ with a significance of $4.4 \sigma$. The data set of 1 ES $2344+514$ can be split into three independent observation periods. The first period P1 ranges from October to December 1997, the second period P2 from August to November 1998. P1 and P2 are separated by a period of non observability of the object from the HEGRA site. Additional observations have been carried out in September 2002 (P3). The data subset P1 shows no evidence for a TeV $\gamma$-ray signal, with a DC significance of $0.3 \sigma$ whereas the second observation period P2 yields a significance of $4.3 \sigma$. In the last observation period P3 an excess on the $2.6 \sigma$ level is found. Tests for burst-like behaviour do not yield statistically significant results. In Table 2, the number of on- and off-source events as well as the corresponding significances are listed for the different data subsamples. In Fig. 2 the distributions of the reconstructed directions for the complete data set $(\mathrm{P} 1+\mathrm{P} 2+\mathrm{P} 3)$ and the data set with the highest significance $(\mathrm{P} 2)$ of $1 \mathrm{ES} 2344+514$ are shown. The observed excess results in a flux of $\Phi(E>0.97 \mathrm{TeV})=(0.60 \pm 0.19) \times 10^{-12}$ photons $\mathrm{cm}^{-2} \mathrm{~s}^{-1}$, corresponding to $(3.3 \pm 1.0) \%$ of the Crab Nebula flux. The errors on the flux level are dominated by the statistics of the measurements.

In contrast to the abovementioned four objects, the jet of the giant radio galaxy M 87 is not aligned to our line of sight which makes it the only non BL Lac type object among the 4 most 
1ES 2344+514

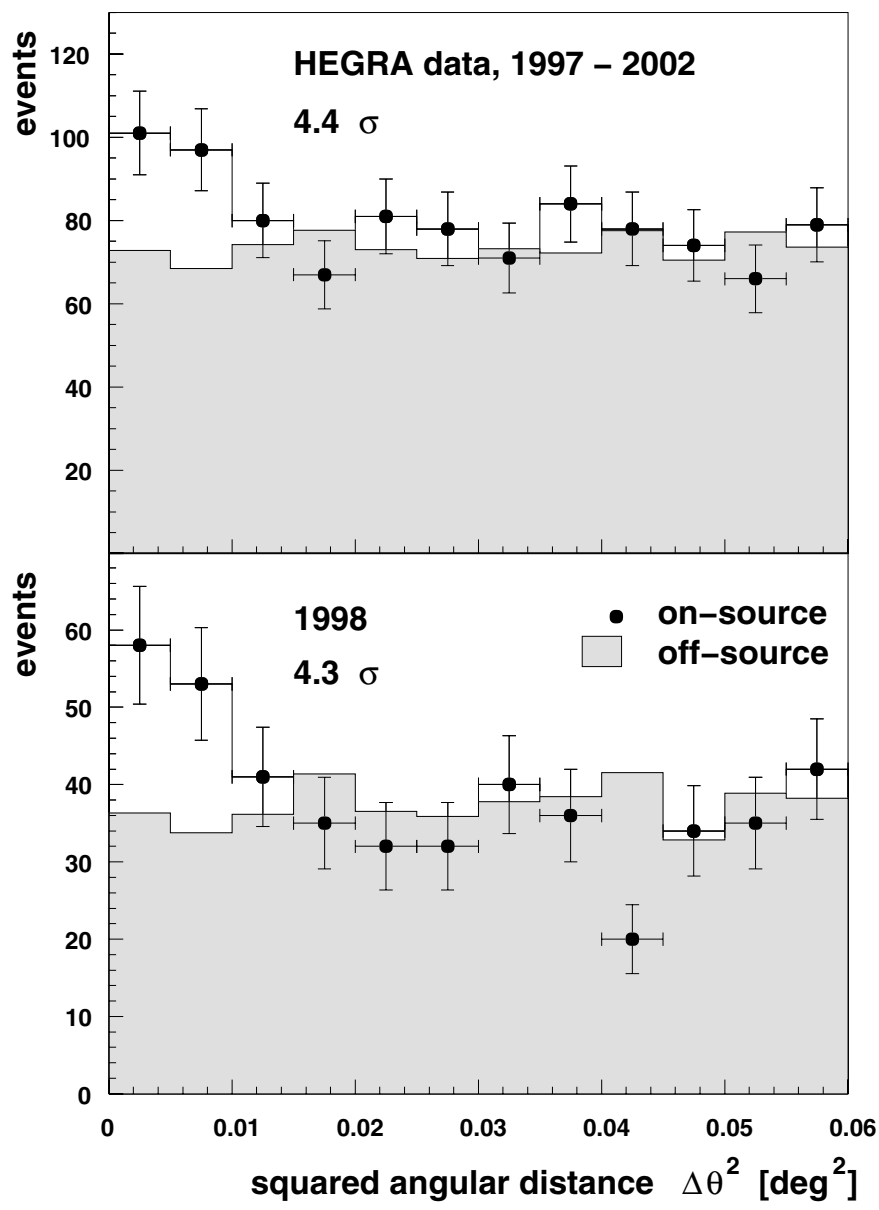

Fig. 2. Distributions of reconstructed squared angular distances to the object direction (see text) of the 1ES $2344+514$ data. The distribution of the on-source events is represented by the data points. The scaled background (off) is shown as a shaded histogram. The upper figure represents the total data set taken between 1997 and 2002 whereas the lower figure shows the 1998 data alone. As can be seen from this figure the excess accumulates essentially in the year 1998.

significant objects of this data sample. The observed excess from the radio galaxy $\mathrm{M} 87$ results in a significance of $3.9 \sigma$ in the present analysis and is consistent to the results of a detailed analysis of the M 87 data, which yield $4.1 \sigma$ and were presented in a dedicated paper (Aharonian et al. 2003b). After further improvements this analysis now yields $4.7 \sigma$ (Götting et al. 2003).

Several objects that were reported to be sources of $\mathrm{TeV} \gamma$-rays by other groups are included in this data set. Among these, the objects 3C 066A (Neshpor et al. 1998) and PKS 2155-304 (Chadwick et al. 1999; Djannati-Ataï et al. 2003) were only observed for a very short time. No excess was found in the HEGRA data of these two objects. A weak excess on the $3 \sigma$ level is found in BL-Lacertae which was observed for $29 \mathrm{~h}$, resulting in a $99 \%$ C.L. upper limit of the order of $28 \%$ of the Crab Nebula flux (see Table 4). This object was reported to be a $\mathrm{TeV} \gamma$-ray emitter by the Crimean Observatory (Neshpor et al. 2001).

\section{Discussion}

Predictions for $\gamma$-ray fluxes in the $\mathrm{GeV} / \mathrm{TeV}$ energy regime have been made for several AGN by Stecker et al. (1996) and Costamante \& Ghisellini (2002). In Table 5, the observed integral fluxes and flux upper limits $(\Phi)$ of several objects of the present data sample are compared to predictions $\left(\Phi_{\text {model }}\right)$ made by these authors and by our own implementation of a parametrization from Fossati et al. (1998). For all objects listed in Table 5, the derived HEGRA upper limits on the integral flux were calculated for an energy threshold of $1 \mathrm{TeV}$ and for different spectral indices, assuming a power law energy spectrum. In the first two columns, the object name and redshift are given. In the third column, predictions made by Stecker et al. (1996) (labeled S), based upon simple scaling arguments, taking into account the Einstein Slew survey sample of BL Lacs, are given. The authors argue that only high frequency peaked BL Lac objects are potential sources of extragalactic TeV $\gamma$-radiation. The observed fluxes of 1ES 1959+650 in its low state and 1ES $2344+514$ are close to the predicted values. The calculated HEGRA upper limits for all other objects in this list exceed the predictions. For some objects (e.g. 1ES 0927+500, $1 \mathrm{ES} 1440+122)$ the predicted flux levels are a factor of 50 to 100 lower than the upper limits from HEGRA. The level of sensitivity necessary to detect such low fluxes within a reasonable time is beyond the capabilities of the HEGRA Cherenkov telescopes. Such low fluxes could only be detected by the experiments of the next generation which have a higher sensitivity and a lower energy threshold.

In Cols. 4 and 5 of Table 5, our results are compared to predictions made by Costamante \& Ghisellini (2002). Two different model predictions are given by these authors. The first prediction is taken from a parametrization of the spectral energy distribution (SED) originally introduced by Fossati et al. (1998) and modified by Donato et al. (2001) and Costamante \& Ghisellini (2002) (hereafter FDC). The second prediction is calculated using an SSC model from Costamante \& Ghisellini (2002) (hereafter CG). Compared to the earlier work of Stecker et al. (1996), CG introduce the new selection criterion of strong radio emission for a $\mathrm{TeV}$ candidate source (arguing that a strong radio emission is a good indicator for non-thermal low energy emission producing seed photons). Furthermore, several other BL Lac samples in addition to the Einstein Slew survey sample were taken into account. The FDC parametrization is rather suitable for predictions of high state $\mathrm{TeV}$ fluxes while the SSC model predictions, designed to fit the known synchrotron part of the SED, are more appropriate for a quiescent state of the $\mathrm{TeV}$ source candidate. Additionally, one has to note that, along with other uncertainities, the absorption of $\mathrm{TeV}$ photons by the extragalactic background radiation field was not accounted for in these models (CG).

In addition to the flux predictions given in $\mathrm{CG}$, we have used our own implementation of the FDC parametrization to 
Table 3. This table summarizes the results of the present analysis for the most significant objects from the sample of 54 AGN observed with the system of stereoscopic Cherenkov telescopes. The number of on- and off-events as well as significance $S$, fluxes in units of the Crab Nebula flux $(F)$ and in units of $10^{-12}$ photons $\mathrm{cm}^{-2} \mathrm{~s}^{-1}(\Phi)$ above the energy threshold $E_{\mathrm{thr}}$ of the observation are given.

\begin{tabular}{|c|c|c|c|c|c|c|}
\hline Object & $\begin{array}{r}N_{\text {on }} \\
{[\#]}\end{array}$ & $\begin{array}{r}\alpha N_{\text {off }} \\
{[\#]}\end{array}$ & $\begin{array}{c}S \\
{[\sigma]}\end{array}$ & $\begin{array}{c}E_{\mathrm{thr}} \\
{[\mathrm{TeV}]}\end{array}$ & $\begin{array}{c}F \\
{[\mathrm{Crab}]}\end{array}$ & $\begin{array}{c}\Phi \\
{\left[10^{-12} \text { photons } \mathrm{cm}^{-2} \mathrm{~s}^{-1}\right]}\end{array}$ \\
\hline 1ES $1959+650$ & 1212 & 454 & 28.0 & 1.32 & $0.06-2.9$ & $0.6-30.5$ \\
\hline H $1426+428$ & 836 & 654 & 6.6 & 0.91 & 0.03 & 0.8 \\
\hline 1ES 2344+514 & 235 & 171 & 4.4 & 0.97 & 0.03 & 0.6 \\
\hline M 87 & 241 & 184 & 3.9 & 0.88 & 0.04 & 0.8 \\
\hline
\end{tabular}

calculate flux predictions for several objects not included in the list of CG (hereafter FDCA). We have additionally included the effect of the absorption of $\mathrm{TeV}$ photons by pair production with the extragalactic background light (EBL). For this purpose we have used a model parametrization of the spectral energy distribution of the EBL (also used in Aharonian et al. 2002b, model 1) adopted from Primack (2001) and designed to be consistent with our observations of known TeV Blazars. The luminosity distance was calculated following Ue-Li Pen (1996). These predictions are compared to upper limits calculated in this work in Col. 6 of Table 5. Differences for some sources between our own implementation of the FDC-parametrization (FDCA) and the implementation by Costamante et al. (2001) (FDC) might be due to the luminosity distance calculation used. In the case of the FDCA parametrization, the predicted flux levels exceed the derived upper limits for those objects printed in boldface in Table 5. Assuming these predictions to hold true for a high state of activity, it can be concluded that these objects were not in a flaring state during the HEGRA observations. However, if we take into account the absorption by the EBL (Col. 7, Table 5, FDCA+EBL) this only remains valid for four objects.

For 1ES 1959+650, the observed flux level during the highest state of emission in the HEGRA data is found to exceed the predicted value by a factor of 3 (FDCA only) to 12 (FDCA+EBL). Given the high variability of the object at this time, prediction and observation can easily be accomodated. The observed flux from $\mathrm{H} 1426+428$ is roughly a factor of 3 below the value predicted by the FDC parametrisation alone. But including the absorption by the EBL the predicted value is much lower than the observed flux (FDCA+EBL). The observed flux level from 1ES 2344+514 is lower than the predicted value by a factor of 30 (FDCA only) and a factor of 8 (FDCA+EBL). However, this object has shown flux levels in the earlier Whipple data which exceeded the flux observed by HEGRA by a factor of 20 , which shows that during HEGRA observations 1ES 2344+514 was indeed not in a flaring state. The SSC model of Costamante \& Ghisellini (2002) predicts flux values well below the observed upper limits. Most of these predicted values would only be detectable with much longer exposure times or with the next generation of Cherenkov telescopes.

\section{Summary}

A data set of 54 Active Galactic Nuclei observed with the HEGRA IACT system between 1996 and 2002 has been analysed. The two objects 1ES $1959+650$ and H 1426+428 have since been well-established as sources of $\mathrm{TeV} \gamma$-radiation and are discussed in Aharonian et al. (2002b, 2003c,a). The evidence for TeV $\gamma$-rays from 1ES $2344+514$ is a confirmation of the detection of this object by the Whipple collaboration. The detection of $\mathrm{TeV} \gamma$-rays from M 87 (Aharonian et al. 2003b) would be, if confirmed, the first detection of photons in the $\mathrm{TeV}$ energy regime from an AGN of an object not commonly classified as a BL Lac object. Upper limits have been derived for all other 50 objects. Table 3 summarizes the results for the most significant excesses seen in this data set. Comparisons with different model predictions indicate that a higher sensitivity is needed to be able to constrain the models.

A further step towards understanding the acceleration mechanisms involved and for the comprehension of the AGN class as a whole, as well as of the absorption by the extragalactic background radiation field, is expected from further observations of AGN and especially BL Lac type objects over a wide range of redshifts with the next generation of Cherenkov telescopes (partly already in operation) and with future instruments. With the reduction of the energy threshold towards $100 \mathrm{GeV}$ the effect of the absorption by the extragalactic background light will decrease for objects located at low redshift. Thus, the uncertainties in the interpretation of observations induced by this effect will be lessened.

Acknowledgements. The support of the German Federal Ministry for Research and Technology BMBF and of the Spanish Research Council CICYT is gratefully acknowledged. G.R. acknownledges receipt of a von Humboldt fellowship. We thank the Instituto de Astrofísica de Canarias (IAC) for the use of the HEGRA site at the Observatorio del Roque de los Muchachos (ORM) and for supplying excellent working conditions on La Palma. This research has made use of the SIMBAD database, operated at CDS, Strasbourg, France and of the NASA/IPAC Extragalactic Database (NED) which is operated by the Jet Propulsion Laboratory, California Institute of Technology, under contract with the National Aeronautics and Space Administration. Useful suggestions by Dr. M. Punch are greatfully acknowledged. 
Table 4. List of all objects of the HEGRA AGN data sample. The J2000 coordinates are given as well as redshift and object type (following Stickel et al. 1994; Padovani \& Giommi 1995; Véron-Cetty \& Véron 2001). The results of the analysis presented here are summarized. In case of the objects deviating significantly from the background expectation the flux is given in units of the Crab Nebula flux. For all other objects upper limits on the integral flux in units of the Crab Nebula flux $\left(F_{\mathrm{UL}}^{99 \%}\right)$ and in units of $10^{-12}$ photons $\mathrm{cm}^{-2} \mathrm{~s}^{-1}\left(\Phi_{\mathrm{UL}}^{99 \%}\right)$ are given.

\begin{tabular}{|c|c|c|c|c|c|c|c|c|c|c|}
\hline Object & $\begin{array}{c}\alpha_{r} \\
\text { (hh mm ss) }\end{array}$ & $\begin{array}{c}\delta \\
(\mathrm{dd} \mathrm{mm} \mathrm{ss})\end{array}$ & $\bar{z}$ & Type & $\begin{array}{c}T \\
{[\mathrm{~h}]}\end{array}$ & $\begin{array}{c}E_{\mathrm{thr}} \\
{[\mathrm{TeV}]}\end{array}$ & $\begin{array}{c}S \\
{[\sigma]}\end{array}$ & $\begin{array}{c}F_{\mathrm{UL}}^{99 \%} \\
{[\mathrm{Crab}]}\end{array}$ & $\begin{array}{c}\Phi_{\mathrm{UL}}^{99 \%} / 10^{-12} \\
{\left[\gamma \mathrm{cm}^{-2} \mathrm{~s}^{-1}\right]}\end{array}$ & $\begin{array}{c}F \\
{[\mathrm{Crab}]}\end{array}$ \\
\hline $1 \mathrm{ES} 0647+250$ & 065046.6 & +250300 & - & $\mathrm{BL}$ & 4.1 & 0.78 & 0.5 & 0.13 & 3.35 & \\
\hline MG 0509+0541 & 050926.0 & +054135 & - & BL & 15.8 & 0.96 & 1.3 & 0.11 & 1.92 & \\
\hline M 87 & 123049.4 & +122328 & 0.004 & $\mathrm{~F} 1$ & 70.0 & 0.88 & 3.9 & & & 0.04 \\
\hline NGC 315 & 005748.9 & +302108 & 0.016 & $\mathrm{~F} 1 / 2$ & 14.6 & 0.86 & -0.3 & 0.05 & 1.03 & \\
\hline NGC 1275 & 031948.2 & +413042 & 0.018 & $\mathrm{~F} 1$ & 87.6 & 0.85 & -0.3 & 0.03 & 0.68 & \\
\hline H $1722+119$ & 172504.5 & +115215 & 0.018 & BL & 5.1 & 0.89 & 1.7 & 0.21 & 4.31 & \\
\hline PKS 2201+04 & 220417.7 & +044003 & 0.028 & S1 & 17.8 & 0.95 & 1.7 & 0.08 & 1.40 & \\
\hline V Zw 331 & 031357.0 & +411537 & 0.029 & BL & 4.1 & 0.87 & -0.2 & 0.09 & 1.93 & \\
\hline NGC 1054 & 024215.0 & +181300 & 0.032 & $\mathrm{G}$ & 57.9 & 0.86 & -1.7 & 0.02 & 0.37 & \\
\hline $3 \mathrm{C} 120$ & 043312.0 & +052115 & 0.033 & $\mathrm{~F} 1$ & 25.4 & 0.93 & -0.7 & 0.05 & 0.86 & \\
\hline NGC 4151 & 121032.7 & +392419 & 0.033 & $\mathrm{~S} 1.5$ & 7.0 & 0.79 & -0.4 & 0.07 & 1.79 & \\
\hline UGC 01651 & 020938.5 & +354751 & 0.037 & $\mathrm{G}$ & 14.3 & 0.79 & 1.3 & 0.07 & 1.62 & \\
\hline UGC 03927 & 073730.0 & +594103 & 0.041 & $\mathrm{~F} 2$ & 6.3 & 1.09 & -2.4 & 0.09 & 1.32 & \\
\hline 1ES 2344+514 & 234704.9 & +514217 & 0.044 & $\mathrm{BL}$ & 72.5 & 0.97 & 4.4 & & & 0.03 \\
\hline Mkn 0180 & 113626.4 & +700927 & 0.046 & BL & 9.8 & 1.50 & -0.6 & 0.12 & 1.09 & \\
\hline 1ES 1959+650 & 195959.9 & +650854 & 0.047 & BL & 163.7 & 1.32 & 28.0 & & & $0.06-2.9$ \\
\hline 3C 371.0 & 180650.7 & +694928 & 0.050 & BL & 5.4 & 1.52 & -0.4 & 0.19 & 1.65 & \\
\hline $4 C+37.11$ & 040549.3 & +380332 & 0.054 & $\mathrm{~S}$ & 6.7 & 0.80 & -2.0 & 0.05 & 1.17 & \\
\hline I Zw 187 & 172818.6 & +501310 & 0.055 & $\mathrm{BL}$ & 16.0 & 0.94 & 1.9 & 0.09 & 1.66 & \\
\hline Cyg-A (3C 405.0) & 195928.5 & +404402 & 0.057 & F2 & 59.0 & 0.91 & -0.2 & 0.03 & 0.64 & \\
\hline 1ES $2321+419$ & 232352.5 & +421055 & 0.059 & BL & 22.3 & 0.89 & -1.6 & 0.03 & 0.67 & \\
\hline 3C 192.0 & 080535.0 & +240950 & 0.060 & $\mathrm{~F} 2$ & 2.9 & 0.93 & 0.3 & 0.20 & 3.78 & \\
\hline $4 C+31.04$ & 011935.0 & +321050 & 0.060 & FR & 3.0 & 0.76 & -0.3 & 0.14 & 3.83 & \\
\hline BL-Lacertae & 220243.3 & +421640 & 0.069 & $\mathrm{BL}$ & 26.7 & 1.10 & 3.0 & 0.28 & 4.10 & \\
\hline 1ES $1741+196$ & 174357.8 & +193509 & 0.083 & BL & 10.2 & 0.94 & 0.3 & 0.07 & 1.41 & \\
\hline $4 C+01.13$ & 051352.5 & +015710 & 0.084 & $\mathrm{~F} 2$ & 7.7 & 1.01 & -0.2 & 0.10 & 1.73 & \\
\hline PKS 2155-304 & 215852.0 & -301332 & 0.116 & $\mathrm{BL}$ & 1.8 & 5.72 & 0.0 & 0.27 & 0.28 & \\
\hline 1ES $1118+424$ & 112048.1 & +421212 & 0.124 & BL & 2.0 & 0.97 & 0.3 & 0.24 & 4.31 & \\
\hline 1ES $0145+13.8$ & 014829.8 & +140219 & 0.125 & BL & 3.2 & 0.87 & 1.1 & 0.06 & 1.37 & \\
\hline $1 \mathrm{H} 0658+595$ & 071030.1 & +590820 & 0.125 & BL & 33.7 & 1.08 & -0.4 & 0.06 & 0.91 & \\
\hline Н $1426+428$ & 142832.5 & +424025 & 0.129 & $\mathrm{BL}$ & 258.5 & 0.91 & 6.6 & & & 0.03 \\
\hline 3C 197.1 & 082132.6 & +470246 & 0.130 & QSO & 15.0 & 0.96 & -0.4 & 0.05 & 0.86 & \\
\hline 1ES $1212+078$ & 121511.2 & +073202 & 0.130 & $\mathrm{BL}$ & 2.4 & 0.92 & -0.6 & 0.17 & 3.24 & \\
\hline 1ES 0806+524 & 080949.2 & +521858 & 0.138 & BL & 1.0 & 1.09 & -0.1 & 0.29 & 4.25 & \\
\hline 1ES 0229+200 & 023248.7 & +2017 17 & 0.139 & BL & 3.0 & 0.92 & 1.0 & 0.17 & 3.25 & \\
\hline RBS 0958 & 111706.3 & +201406 & 0.139 & BL & 3.8 & 0.85 & 2.7 & 0.28 & 6.23 & \\
\hline 1ES $1255+244$ & 125732.0 & +241239 & 0.140 & BL & 5.9 & 0.94 & 0.1 & 0.12 & 2.16 & \\
\hline MS1019.0+5139 & 102211.0 & +512400 & 0.141 & S & 17.5 & 0.92 & 0.1 & 0.07 & 1.35 & \\
\hline 1ES $0323+022$ & 032613.9 & +022514 & 0.147 & BL & 14.3 & 1.00 & -1.5 & 0.04 & 0.71 & \\
\hline OQ 530 & 141946.6 & +542314 & 0.152 & $\mathrm{BL}$ & 9.4 & 1.12 & 0.4 & 0.10 & 1.41 & \\
\hline $3 C 273.0$ & 122906.7 & +020308 & 0.158 & FR & 12.2 & 1.15 & -0.3 & 0.09 & 1.25 & \\
\hline $1 \mathrm{ES} 1440+122$ & 144248.4 & +120039 & 0.162 & $\mathrm{BL}$ & 13.1 & 0.92 & -0.9 & 0.08 & 1.49 & \\
\hline PKS 0829+046 & 083148.9 & +04 2939 & 0.180 & BL & 18.0 & 1.00 & 0.5 & 0.06 & 0.96 & \\
\hline PG $1218+304$ & 122122.0 & +301037 & 0.182 & BL & 3.9 & 0.84 & -0.3 & 0.12 & 2.67 & \\
\hline 1ES $0347-121$ & 034923.0 & -115926 & 0.185 & BL & 1.9 & 1.46 & 1.8 & 0.56 & 5.14 & \\
\hline 1ES $0927+500$ & 093037.6 & +495024 & 0.186 & BL & 13.3 & 0.94 & 0.2 & 0.06 & 1.08 & \\
\hline PKS $2254+074$ & 225717.3 & +074312 & 0.190 & $\mathrm{BL}$ & 16.3 & 0.90 & -0.5 & 0.05 & 0.99 & \\
\hline MS0317.0+1834 & 031951.9 & +184535 & 0.190 & BL & 2.7 & 0.80 & -0.5 & 0.12 & 2.96 & \\
\hline 1ES $1011+496$ & 101504.2 & +492600 & 0.200 & $\mathrm{BL}$ & 2.0 & 1.02 & -1.3 & 0.11 & 1.80 & \\
\hline 1ES $0120+340$ & 012308.9 & +342050 & 0.272 & BL & 18.9 & 0.83 & -1.2 & 0.04 & 0.87 & \\
\hline $2 \mathrm{E} 0414+0057$ & 041652.5 & +010523 & 0.287 & BL & 4.5 & 1.01 & 0.6 & 0.13 & 2.16 & \\
\hline S5 $0716+714$ & 072153.4 & +712036 & 0.300 & BL & 1.7 & 1.58 & 0.7 & 0.38 & 3.13 & \\
\hline $3 \mathrm{C} 066 \mathrm{~A}$ & 022239.6 & +430207 & 0.444 & $\mathrm{BL}$ & 1.3 & 0.85 & -0.2 & 0.17 & 3.87 & \\
\hline PKS 0219-164 & 022201.0 & -161516 & 0.698 & $\mathrm{BL}$ & 1.7 & 1.78 & -1.7 & 0.27 & 1.85 & \\
\hline
\end{tabular}


Table 5. Comparison of upper limits and fluxes $(\Phi)$ derived in this work with predictions $\left(\Phi_{\text {model }}\right)$ made by Stecker et al. (1996) (S), Costamante \& Ghisellini (2002) (CG, FDC) and with our own implementation of a parametrization from Fossati et al. (1998) (FDCA) and including the absorption by the extragalactic background light (FDCA+EBL). For 1ES 0647+250 a redshift of 0.200 was assumed. All fluxes are given in units of $10^{-12}$ photons $\mathrm{cm}^{-2} \mathrm{~s}^{-1}$. The $99 \%$ C.L. upper limits were extrapolated to a fixed energy threshold of $1 \mathrm{TeV}$ assuming a power law energy spectrum for 3 differential spectral indices $\alpha=2.0,2.5,3.0$. For the objects 1ES 1959+650, H 1426+428 and 1ES $2344+514$ fluxes are given. Objects printed in boldface show upper limits below some of the predicted values.

\begin{tabular}{|c|c|c|c|c|c|c|c|c|c|}
\hline \multirow[t]{3}{*}{ Object } & \multirow[t]{3}{*}{$z$} & \multicolumn{5}{|c|}{$\begin{array}{c}\Phi_{\text {model }} / 10^{-12} \\
\gamma \mathrm{cm}^{-2} \mathrm{~s}^{-1}\end{array}$} & \multirow{2}{*}{\multicolumn{3}{|c|}{$\begin{array}{c}\Phi / 10^{-12} \\
\gamma \mathrm{cm}^{-2} \mathrm{~s}^{-1} \\
\text { spectral index } \alpha\end{array}$}} \\
\hline & & \multirow[t]{2}{*}{$\mathrm{S}$} & \multirow{2}{*}{$\begin{array}{l}\text { FDC } \\
\text { from } \mathrm{CG}\end{array}$} & \multirow[t]{2}{*}{$\mathrm{CG}$} & \multirow[t]{2}{*}{ FDCA } & \multirow[t]{2}{*}{ FDCA+EBL } & & & \\
\hline & & & & & & & 2.0 & 2.5 & 3.0 \\
\hline H 1722+119 & 0.018 & - & 35.2 & 0.01 & 36.98 & 20.53 & $<3.84$ & $<3.62$ & $<3.41$ \\
\hline 1ES $2344+514$ & 0.044 & 0.80 & - & - & 18.58 & 4.72 & $=0.58$ & $=0.57$ & $=0.56$ \\
\hline Mkn 180 & 0.046 & - & - & - & 19.00 & 4.56 & $<1.64$ & $<2.00$ & $<2.45$ \\
\hline 1ES $1959+650$ & 0.047 & 2.30 & 17.4 & - & 18.01 & 4.20 & & $(1.0-51$ & \\
\hline 3C 371.0 & 0.050 & - & - & - & 12.41 & 2.80 & $<2.51$ & $<3.09$ & $<3.81$ \\
\hline I Zw 187 & 0.055 & 0.59 & - & - & 12.40 & 2.25 & $<1.56$ & $<1.51$ & $<1.47$ \\
\hline 1ES $2321+419$ & 0.059 & - & - & - & 4.85 & 0.75 & $<0.60$ & $<0.56$ & $<0.53$ \\
\hline BL Lacertae & 0.069 & - & - & - & 1.66 & 0.22 & $<4.51$ & $<4.73$ & $<4.96$ \\
\hline 1ES $1741+196$ & 0.083 & 0.35 & 8.4 & 0.1 & 7.10 & 0.55 & $<1.33$ & $<1.29$ & $<1.25$ \\
\hline PKS 2155-304 & 0.116 & 0.88 & - & - & 2.40 & 0.07 & $<1.60$ & $<3.83$ & $<9.16$ \\
\hline 1ES $1118+424$ & 0.124 & 0.18 & - & - & 2.36 & 0.05 & $<4.18$ & $<4.12$ & $<4.06$ \\
\hline 1ES $0145+138$ & 0.125 & 0.26 & - & - & 1.16 & 0.02 & $<1.19$ & $<1.11$ & $<1.04$ \\
\hline $1 \mathrm{H} 0658+595$ & 0.125 & - & - & - & 2.49 & 0.05 & $<0.98$ & $<1.02$ & $<1.06$ \\
\hline H $1426+428$ & 0.129 & - & - & - & 2.32 & 0.04 & & $=0.69$ & \\
\hline 1ES $1212+078$ & 0.130 & 0.03 & - & - & 2.74 & 0.05 & $<2.98$ & $<2.86$ & $<2.74$ \\
\hline 1ES 0806+524 & 0.138 & - & 2.7 & - & 1.85 & 0.03 & $<4.63$ & $<4.84$ & $<5.05$ \\
\hline 1ES $0229+200$ & 0.139 & 0.11 & 2.1 & 0.04 & 2.25 & 0.03 & $<2.99$ & $<2.87$ & $<2.75$ \\
\hline RBS 0958 & 0.139 & - & 2.8 & - & 2.42 & 0.03 & $<5.30$ & $<4.88$ & $<4.50$ \\
\hline 1ES $1255+244$ & 0.140 & 0.34 & - & - & 1.12 & 0.01 & $<2.03$ & $<1.97$ & $<1.91$ \\
\hline 1ES $0323+022$ & 0.147 & 0.15 & 1.8 & - & 1.97 & 0.02 & $<0.71$ & $<0.71$ & $<0.71$ \\
\hline OQ 530 & 0.152 & - & - & - & 0.23 & $3 \times 10^{-3}$ & $<1.58$ & $<1.67$ & $<1.77$ \\
\hline 1ES $1440+122$ & 0.162 & 0.03 & 2.0 & 0.1 & 1.69 & 0.01 & $<1.37$ & $<1.31$ & $<1.26$ \\
\hline PKS 0829+046 & 0.180 & - & - & - & 0.03 & $2 \times 10^{-4}$ & $<0.96$ & $<0.96$ & $<0.96$ \\
\hline PG 1218+304 & 0.182 & - & 1.5 & - & 1.24 & $5 \times 10^{-3}$ & $<2.24$ & $<2.06$ & $<1.88$ \\
\hline 1ES 0347-121 & 0.185 & 0.08 & - & - & 0.83 & $3 \times 10^{-3}$ & $<7.50$ & $<9.07$ & $<10.96$ \\
\hline 1ES $0927+500$ & 0.186 & 0.02 & - & - & 1.06 & $3 \times 10^{-3}$ & $<1.02$ & $<0.98$ & $<0.95$ \\
\hline PKS 2254+074 & 0.190 & - & - & - & 0.06 & $2 \times 10^{-4}$ & $<0.89$ & $<0.85$ & $<0.80$ \\
\hline MS $0317.0+1834$ & 0.190 & - & - & - & 1.00 & $3 \times 10^{-3}$ & $<2.37$ & $<2.12$ & $<1.89$ \\
\hline $1 \mathrm{ES} 0647+250$ & 0.200 & - & 1.2 & - & 0.83 & $2 \times 10^{-3}$ & $<2.61$ & $<2.31$ & $<2.04$ \\
\hline 1ES $1011+496$ & 0.200 & - & 0.2 & - & 0.29 & $7 \times 10^{-4}$ & $<1.84$ & $<1.85$ & $<1.87$ \\
\hline 1ES $0120+340$ & 0.272 & - & 0.6 & - & 0.37 & $8 \times 10^{-5}$ & $<0.72$ & $<0.66$ & $<0.60$ \\
\hline $2 \mathrm{E} 0414+0057$ & 0.287 & - & - & - & 0.22 & $3 \times 10^{-5}$ & $<2.18$ & $<2.19$ & $<2.20$ \\
\hline S5 0716+714 & 0.300 & - & - & - & 0.21 & $2 \times 10^{-5}$ & $<4.95$ & $<6.22$ & $<7.81$ \\
\hline $3 \mathrm{C} 66 \mathrm{~A}$ & 0.444 & - & - & - & 0.11 & $1 \times 10^{-7}$ & $<3.29$ & $<3.03$ & $<2.80$ \\
\hline PKS 0219-164 & 0.698 & - & - & - & 0.04 & $5 \times 10^{-12}$ & $<3.29$ & $<4.39$ & $<5.86$ \\
\hline
\end{tabular}

\section{References}

Aharonian, F., Daum, A., Hermann, G., et al. 1997, A\&A, 327, L5 Aharonian, F., Akhperjanian, A. G., Barrio, J. A., et al. 1999a, A\&A, 349,11

Aharonian, F., Akhperjanian, A. G., Barrio, J. A., et al. 1999b, A\&A, 349,29
Aharonian, F., Akhperjanian, A. G., Andronache, M., et al. 1999c, A\&A, 350, 757

Aharonian, F. A. 2000, New Astron., 5, 377

Aharonian, F., Akhperjanian, A. G., Barrio, J. A., et al. 2001a, A\&A, 366,62

Aharonian, F., Akhperjanian, A. G., Barrio, J. A., et al. 2001b, A\&A, 366,746 
Aharonian, F. 2001, Proc. of the 27th ICRC, Hamburg, Highlight Papers, 250 [arXiv: astro-ph/0112314]

Aharonian, F. A. 2002, MNRAS, 332, 215

Aharonian, F., Akhperjanian, A. G., Beilicke, M., et al. 2002a, A\&A, 393, 89

Aharonian, F., Akhperjanian, A., Barrio, J., et al. 2002b, A\&A, 384, L23

Aharonian, F., Akhperjanian, A. G., Beilicke, M., et al. 2003a, A\&A, 403, 523

Aharonian, F., Akhperjanian, A. G., Beilicke, M., et al. 2003b, A\&A, 403, L1

Aharonian, F., Akhperjanian, A. G., Beilicke, M., et al. 2003c, A\&A, 406, L9

Bai, J. M., \& Lee, M. G. 2002, ApJ, 549, L173

Baltz, E. A., Briot, C., Salati, P., et al. 2000, Phys. Rev. D, 61, 023514

Bicknell, G. V., \& Begelmann, M. C. 1996, ApJ, 467, 597

Biermann, P. L., Ahn, E., Medina-Tanco, G., \& Stanev, T. 2000, Nucl. Phys. B Proc. Suppl., 87, 417 [arXiv: astro-ph/9911123], [arXiv: astro-ph/0008063]

Bradbury, S. M., Deckers, T., Petry, D., et al. 1997, A\&A, 320, L5

Catanese, M., Akerlof, C. M., Badran, H. M., et al. 1998, ApJ, 501, 616

Chadwick, P. M., Lyons, K., McComb, T. J. L., et al. 1999, ApJ, 513, 161

Costamante, L., Ghisellini, G., Giommi, P., et al. 2001, A\&A, 371, 512

Costamante, L., \& Ghisellini, G. 2002, A\&A, 384, 56

Daum, A., Hermann, G., Hess, M., et al. 1997, Astropart. Phys., 8, 1

de la Calle Perez, I., Bond, I. H., Boyle, P. J., et al. 2003, Proc. of the 28th ICRC, Tsukuba, 5, 2571

Djannati-Ataï, A., Piron, F., Barrau, A., et al. 1999, A\&A, 350, 17

Djannati-Ataï, A., Khelifi, B., Vorobiov, S., et al. 2002, A\&A, 391, L25

Djannati-Ataï, A. 2003, Proc. of the 28th ICRC, Tsukuba, 5, 2575

Donato, D., Ghisellini, G., Tagliaferri, G., \& Fossati, G. 2001, A\&A, 375,739

Elvis, M., Plummer, D., Schachter, J., et al. 1992, ApJS, 80, 257

Fossati, G., Marashi, L., Celotti, A., Comastri, A., \& Ghisellini, G. 1998, MNRAS, 299, 433

Ginzburg, V. L., \& Syrovatskii, S. I. 1965, ARA\&A, 3, 297

Giommi, P., Padovani, P., Perlman, E., et al. 2000, MNRAS, 317, $743 \mathrm{G}$

Götting, N., \& the HEGRA collaboration 2003, to appear in the Proc. of the EPS 2003 conf., Aachen, also [arXiv: astro-ph/0310308]

Helene, O. 1983, Nucl. Instr. Meth., 212, 319

Hofmann, W., Jung, I., Konopelko, A., et al. 1999, Astropart. Phys., 12,135

Horan, D., Badran, H. M., Bond, I. H., et al. 2002, ApJ, 571, 753

Horan, D., Catanese, M. A., Bond, I. H., et al. 2003, Proc. of the 28th ICRC, Tsukuba, 5, 2567
Jones, T. W., O’Dell, S. L., \& Stein, W. A. 1974, ApJ, 188, 353

Kolmogorov, A. N. 1933, Giornale Istituto Italiano Attuari, 4, 83

Konopelko, A., Hemberger, M., Aharonian, F., et al. 1999a, Astropart. Phys., 10, 275

Konopelko, A., Kettler, J., \& the HEGRA Collaboration 1999b, Proc. of the 26th ICRC, Salt Lake City, 3, 426

Krawczynski, H., Sambruna, R., Kohnle, A., et al. 2001, ApJ, 559, 187

Li, T., \& Ma, Y. 1983, ApJ, 272, 317

Mannheim, K. 1993, A\&A, 269, 67

Mirzoyan, R., Kankanian, R., Krennrich, F., et al. 1994, Nucl. Instr. Meth. A, 351, 513

Mücke, A., \& Protheroe, R. J. 2001, Astropart. Phys., 15, 121

Neshpor, Y. I., Stepanyan, A. A., Kalekin, O. P., et al. 1998, Astron. Lett., 24, 134

Neshpor, Y. I., Chalenko, N. N., Stepanian, A. A., et al. 2001, Astron. Rep., 45, 249

Nishiyama, T., Chamoto, N., Chikawa, M., et al. 1999, Proc. of the 26th ICRC, Salt Lake City, 3, 370

Nikishov, A. I. 1962, Sov. Phys. JETP, 14, 393

Padovani, P., \& Giommi, P. 1995, MNRAS, 277, 1477

Petry, D., Bradbury, S. M., Konopelko, A., et al. 1996, A\&A, 311, L13

Piron, F., Djannati-Ataï, A., Punch, M., et al. 2001, A\&A, 358, 895

Prahl, J. 1999 [arXiv: astro-ph/9909399]

Protheroe, R. J., Donea, A.-C., \& Reimer, A. 2003, Astropart. Phys., 19,559

Primack, J. R., Somerville, R. S., Bullock, J. S., \& Devriendt, J. E. G. 2001, High Energy Gamma-Ray Astronomy, AIP Conf. Proc., 558, 463

Pühlhofer, G., Bolz, O., Götting, N., et al. 2003, Astropart. Phys., 20, 267

Punch, M., Akerlof, C. W., Cawley, M. F., et al. 1992, Nature, 160, 477

Quinn, J., Akerlof, C. W., Biller, S., et al. 1996, ApJ, 456, L83

Rees, M. J. 1967, MNRAS, 135, 345

Rees, M. J. 1984, ARA\&A, 22, 471

Reimer, A., Protheroe, R. J., \& Donea, A.-C. 2003, Proc. of the 28th ICRC, Tsukuba, 5, 2631

Sambruna, R., Aharonian, F. A., Krawczynski, H., et al. 2000, ApJ, 538, 127

Schachter, J. F., Stocke, T. J., Perlman, E., et al. 1993, ApJ, 412, 541

Pohl, M., \& Schlickeiser, R. 2000, A\&A, 354, 395

Sikora, M. 2000, AIP Conf. Proc., 558, 275

Stecker, F. W., de Jager, O. C., \& Salamon, M. H. 1996, ApJ, 473, L75

Stickel, M., Meisenheimer, K., \& Kuehr, H. 1994, A\&AS, 105, 211

Tinyakov, P. G., \& Tkachev, I. I. 2001, JETP Lett., 74, 445

Ue-Li Pen 1996, A\&AS, 120, 49

Urry, C. M., \& Padovani, P. 1995, PASP, 107, 803

Véron-Cetty, M. P., \& Véron, P. 2001, A\&A, 374, 92 